IRSTI 29.19.21

https://doi.org/10.26577/phst-2019-2-p10

\title{
Polymorphic phase transitions and recombination luminescence in ammonium halide crystals
}

\author{
T.A. Koketayev and A.K. Tussupbekova* \\ Karaganda State University named after Y.A. Buketov, \\ 100028, 28, Universitetskaya str., Karagandy, Kazakhstan \\ "e-mail adress: tussupbekova.ak@gmail.com
}

\begin{abstract}
Spectral-luminescent properties of ammonium halide crystals were studied in this work. The properties of the peak of thermally stimulated luminescence (TSL) in the region of the phase transition temperature were studied and the basic laws were established. A sequential thermal annealing isolated the TSL peak with a maximum at $240 \mathrm{~K}$ for ammonium chloride activated with rare-earth ions (RI). It was found that an order-disorder phase transition occurs for activated RI of ammonium chloride at a temperature of $240 \mathrm{~K}$. An appearance of TSL peak with a maximum at $240 \mathrm{~K}$ causes because of exposure to ionizing radiation upon activation of ammonium halides by thallium and tin ions. This phenomenon is observed when ammonium halides are activated by europium, ytterbium, neodymium, praseodymium, copper, cadmium and potassium ions. The introduction of impurity homologous anions into the crystal lattice does not give a similar effect. Therefore, it can be argued that observed phenomenon is associated with the presence of impurities in the cationic sublattice and its appearance does not depend on the parameters of the impurity center. On the one hand, the TSL peak is not impurity, since its position is determined by the properties of matrix and on the other hand, its appearance is associated only with substitution ions in the cationic sublattice. The obtained results allow studying the crystals response under exposition to ionizing radiation.
\end{abstract}

Key words: luminescence, radiation-stimulated processes, crystal, defects.

PACS numbers: $61.10 . \mathrm{Ht}, 61.50 . \mathrm{Ks}, 61.72 .-\mathrm{y}, 61.80 .-\mathrm{x}$.

\section{Introduction}

Investigations of the spectral-luminescent properties of ammonium halide crystals activated by various metallic ions have established that phase transitions cause abrupt changes in the parameters of the luminescence centers. The most significant changes are observed in the frequency of active vibrations of impurity ions, electron-phonon interaction, mean free path and activation energy of exciton migration.

In crystals with complex anions and cations, radiation-stimulated processes have a number of specific features. Radiation defects can conditionally be divided into primary and secondary defects. Primary defects arise as a result of exposure to ionizing radiation, secondary defects appear during the transformation of primary defects as a result of migration of radiolysis products. Recombination luminescence is also a consequence of the migration of radiolysis products. It is obvious that the thermal activation of migration and the migration of radiation defects themselves are structurally sensitive. Therefore, polymorphic phase transitions should have a significant effect on the course of radiation-stimulated processes. In this paper, we consider a number of phenomena in irradiated crystals due to rearrangement of the crystal lattice [1-15].

\section{Experiment}

Ammonium halide crystals in the temperature range of 80-300 K have an order-disorder polymorphic phase transition. Figure 1 shows, by way of example, the curves of thermally stimulated luminescence for pure (1) and activated with $\mathrm{Tl}^{+}(2)$ and $\mathrm{Sn}^{2+}$ (3) ions for a crystal of ammonium chloride. In an unactivated crystal, there are two peaks of thermally stimulated luminescence in the region of $110-120 \mathrm{~K}$ 
and $160-180 \mathrm{~K}$. The first peak of the recombination luminescence is associated with the thermal activation of the migration of Vc centers. From an analysis of the results of our studies of thermostimulated cur- rents and thermal bleaching of radiation-induced absorption bands and published data, it was established that the second TSL peak is due to the decay of defects of the $\mathrm{NH}_{3}^{+}$type.

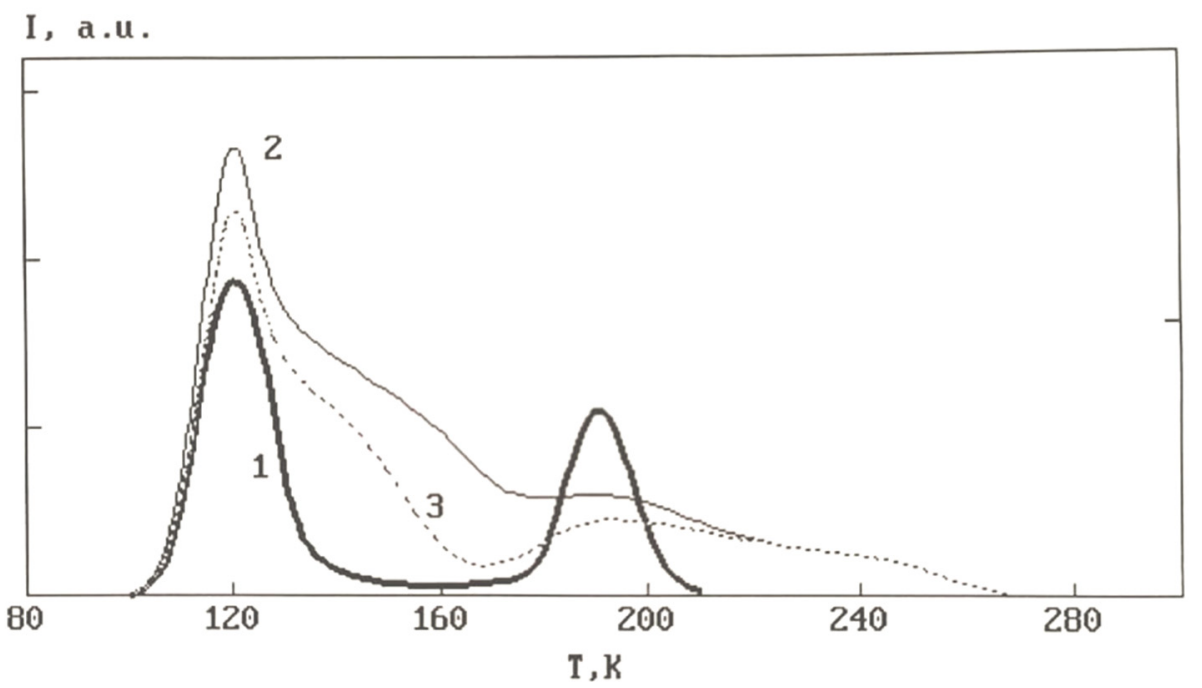

Figure 1 - TSL curves of pure (1) and doped with thallium (2) and tin (3) ions $\mathrm{NH}_{4} \mathrm{Cl}$

When $\mathrm{NH}_{4} \mathrm{Cl}$ crystals are activated by $\mathrm{Tl}^{+}$or $\mathrm{Sn}^{+}$ ions, in addition to the TSL activator peaks, a glow appears in the region of $200-250 \mathrm{~K}$ in the form of a "shoulder" of a high-temperature peak. Similar TSL curves with the same activators are also observed in $\mathrm{NH}_{4} \mathrm{Br}$ crystals (Figure 2).

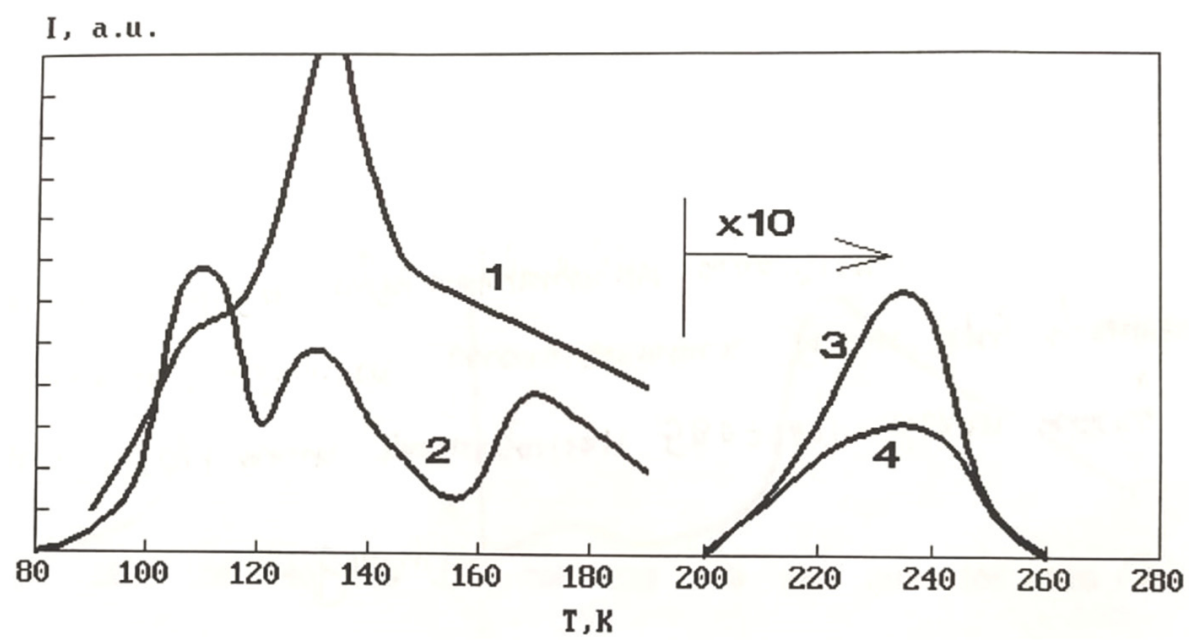

Figure 2 - TSL curves of $\mathrm{NH}_{4} \mathrm{Br}$ crystals activated by thallium (1) and tin ions (2) and after thermal annealing at 190-200 K, respectively (3) and (4) 
For activated RI of ammonium chloride, a TSL peak with a maximum at $240 \mathrm{~K}$ was isolated by sequential thermal annealing (Figure 2). It is known that an order-disorder phase transition occurs at this temperature. Therefore, as a result of the activation of ammonium halides by thallium and tin ions after exposure to ionizing radiation, a TSL peak appears, the maximum of which is linked to the phase transition temperature.

It was established in the work that a similar phenomenon is observed upon activation of ammonium halides by europium, ytterbium, neodymium, praseodymium, copper, cadmium and potassium ions. Thus, regardless of the nature of the impurity ions, a TSL peak appears in the region of the phase transition temperature. The introduction of impurity homologous anions into the crystal lattice does not give a similar effect. Therefore, it can be argued that this phenomenon is associated with the presence of impurities in the cationic sublattice. The appearance of this phenomenon does not depend on the parameters of the impurity center. On the one hand, the TSL peak under study is not impurity, since its position is determined by the properties of the matrix; on the other hand, its appearance is associated only with substitution ions in the cationic sublattice. Studying the properties of the TSL peak in the region of the phase transition temperature, the following basic laws were established:

1. The high-temperature "shoulder" in the region preceding the temperature of the order - disorder structural phase transition arises only in activated and irradiated crystals. Checking the occurrence of this phenomenon due to pre-radiation defects did not give positive results;

2. The appearance of recombination luminescence in the phase transition region is independent of the electronic and charge state of the impurity. The impurity ion must be a substitutional ion in the cationic sublattice.

3. Within the accuracy of the TSL method, the activation energy of the peak extracted by thermal annealing does not depend on the nature of the cationic impurity ion.

4. The spectral composition of the radiation of the high-temperature peak of the matrix and in the phase transition region are qualitatively the same. It consists of a band of activator luminescence and radiation with a maximum of $2.5 \mathrm{eV}$. In the $\mathrm{NH}_{4} \mathrm{Cl}-$ $\mathrm{K}^{+}$crystal, there is radiation in the region of $3.8 \mathrm{eV}$.
However, it cannot be attributed to an activator, because it is optically inactive. It was shown that the 3.8 $\mathrm{eV}$ band can be attributed to a near-activator exciton.

5. The dependences of the accumulated light sum in the studied peak on the exposure dose and concentration reveal the relationship of this phenomenon with impurity ions.

Earlier, we proposed a recombination luminescence model related to structural features and dynamics of $\mathrm{NH}_{3}^{+}$defects $[12,16]$. Its reorientation in the $180 \mathrm{~K}$ region leads to perturbation of the nearest cation and the proton from it can pass into the anionic sublattice, which leads to the launch of recombination mechanisms. Assuming that in activated crystals, cationic impurity ions "freeze" the reorientation of $\mathrm{NH}_{3}^{+}$ defects, the recombination mechanism is triggered by the reorientation of the ammonium ions $\mathrm{NH}_{3}^{+}$themselves. This allows us to explain all the established patterns. Upon recombination of the $\mathrm{NH}_{3}^{+}$ion with hydrogen, a mobile hole appears in the anion sublattice. Its migration and interaction with electronic centers leads to the transfer of energy to impurity ions. An activator glow appears. In this temperature range, the luminescence of the excitons themselves is quenched, etc.

It was experimentally established that pure ammonium halides have a "memory" of previous exposure. In figure 3 shows the corresponding result. Curve 1 - TSL of inactive $\mathrm{NH}_{4} \mathrm{Br}$. Curve 2 - TSL of $\mathrm{NH}_{4} \mathrm{Br}$, measured after the first exposure, heating to $300 \mathrm{~K}$, cooling to $80 \mathrm{~K}$, and re-exposure. It is seen that in this case a "shoulder" appears, which is characteristic of an activated crystal.

It was found that AGA have another TSL peak in the region of $360-380 \mathrm{~K}$, depending on the anion. Its study is difficult, since ammonium halides are sublimated when heated. In view of the foregoing, it can be argued that in AGA this high-temperature TSL peak is associated with a defect in the cationic sublattice. One of all known defects is the hydrazine ion $\mathrm{N}_{2} \mathrm{H}_{4}^{+}$. The crystals "remember" about the previous exposure when stored at room temperature for about 20 hours, which is consistent with the literature on EPR on the stability of hydrazine ions. Thermal annealing of the TSL peak above $300 \mathrm{~K}$ leads to the disappearance of the "memory" effect. Thus, the features of recombination luminescence in the region of the phase transition temperature made it possible to establish a defect, the decay of which is responsible for the high-temperature peak of TSL. 


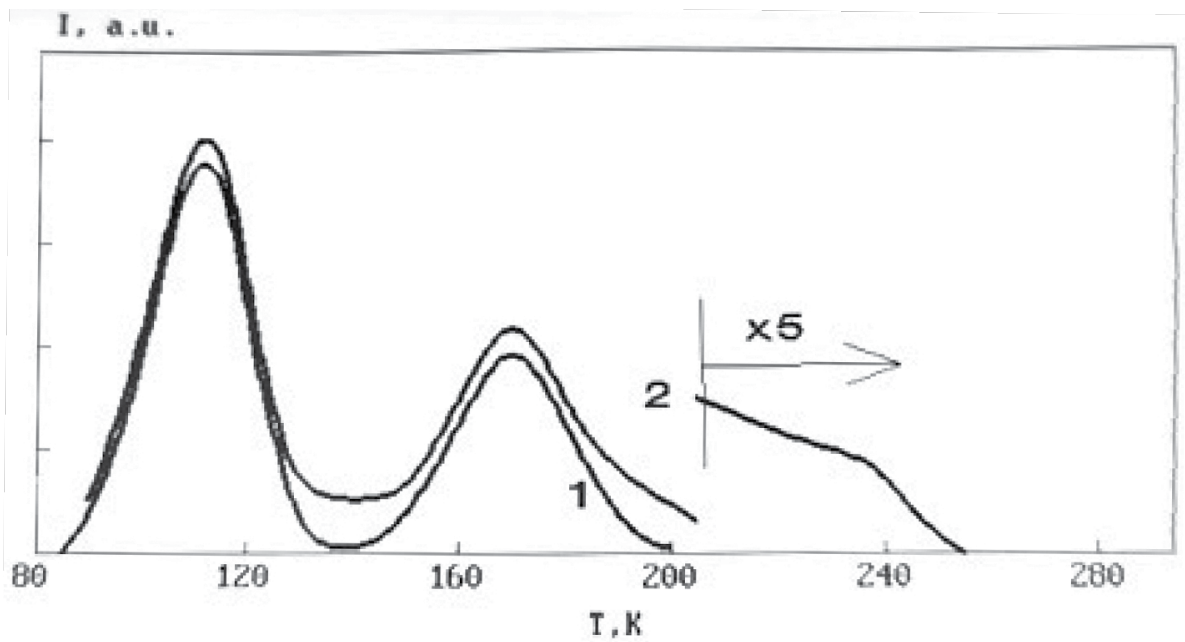

Figure 3 - TSL curves of $\mathrm{NH}_{4} \mathrm{Br}$ pure (1) and after repeated irradiation (2)

$\mathrm{LiKSO}_{4}$ crystals have in the region of $80-300 \mathrm{~K}$ two polymorphic phase transitions at $180 \mathrm{~K}$ and $250 \mathrm{~K}$ in the heating mode. Low-temperature - the first kind and comes with the release of heat, the second - the second kind [13, 17-19].

After irradiation with $\mathrm{x}$-ray quanta at liquid nitrogen temperature, the spectra of thermally stimulated luminescence were measured. In figure 4 shows the TSL curve for the $\mathrm{LiKSO}_{4}$ crystal. A rather complicated picture was obtained, having luminescence maxima at $125 \mathrm{~K}, 150 \mathrm{~K}, 205 \mathrm{~K}, 260 \mathrm{~K}$. In addition, an increase in luminescence is observed in the $170 \mathrm{~K}-180 \mathrm{~K}$ region, accompanied by stochastic outbursts of luminescence intensity. The last peak of the luminescence is complex, since there is a "shoulder" on the high-temperature wing, which indicates the presence of another TSL peak with a lower light sum and strongly overlapping with luminescence at $260 \mathrm{~K}$.

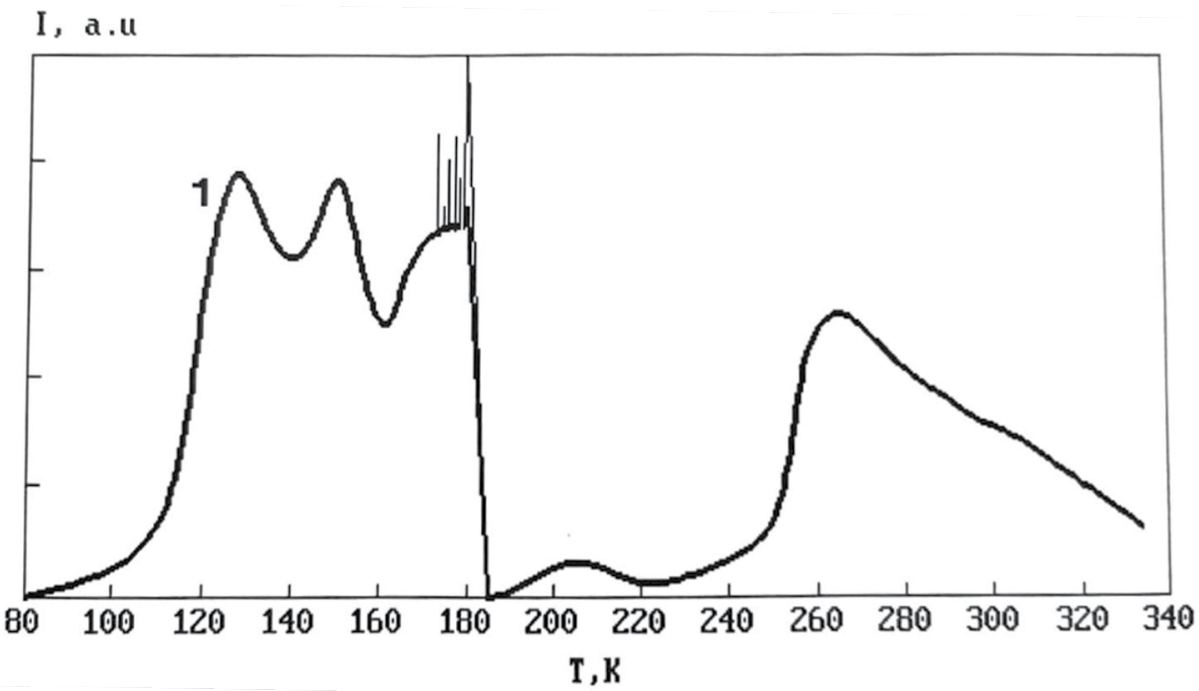

Figure 4 - TSL crystal curve of $\mathrm{LiKSO}_{4}$.

In figure 5 shows the TSL curve of mixed lithium potassium sulfate after irradiation at $80 \mathrm{~K}$, slow heating to a temperature not higher than 175
$\mathrm{K}$, followed by cooling. It is seen that the thermal annealing of the low-temperature TSL peaks leads to the disappearance of a weak glow in the $205 \mathrm{~K}$ 
region. Using the method of subsequent thermal annealing, it was shown that the same recombination process is responsible for the luminescence in the regions of $170-180 \mathrm{~K}$ and $205 \mathrm{~K}$. The appearance of a recombination luminescence peak at $205 \mathrm{~K}$ depends on the heating rate. With a slow rise in temperature, it disappears. It is natural to associate the separation of one peak into two with an abnormal decrease in the glow intensity of the low-temperature component with a polymorphic phase transition at $180 \mathrm{~K}$. It is a first-order transition. The observed anomalies indicate that when the structure of the crystal lattice changes, the activation energy of recombination luminescence increases.

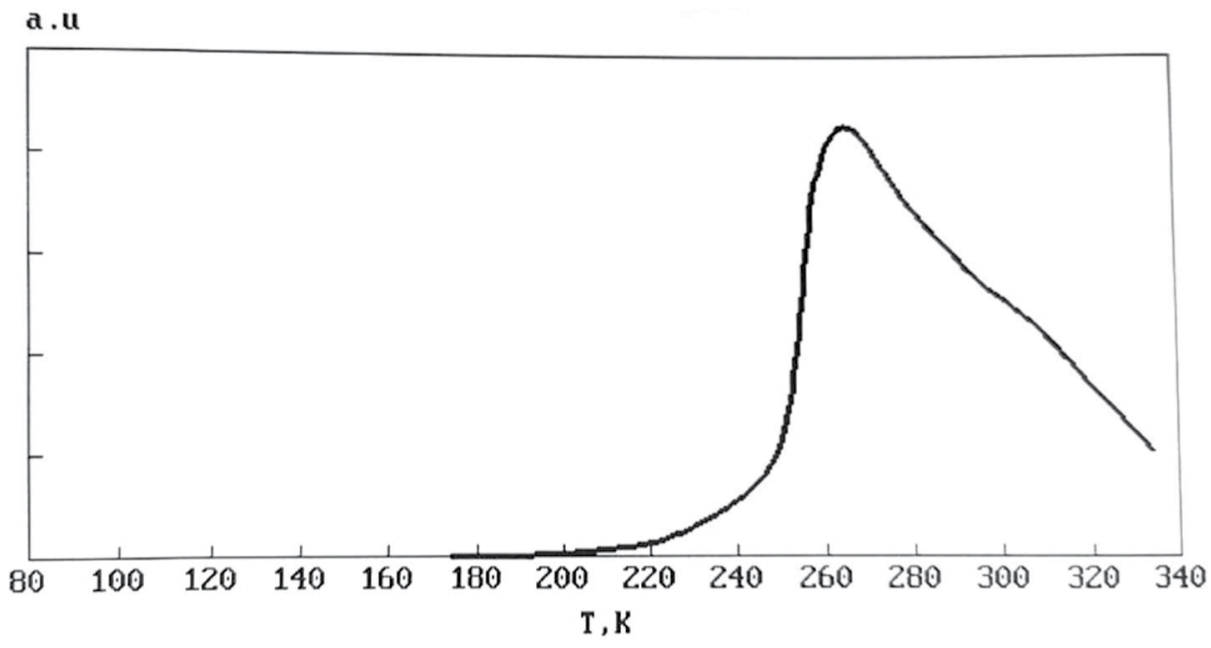

Figure 5 - TSL curve of $\mathrm{LiKSO}_{4}$ crystal after thermal annealing at $175 \mathrm{~K}$

Stochastic emissions in the region of $170-180 \mathrm{~K}$ (see Figure 4) are repeated from experiment to experiment. Their number, intensity, and temperature position of an individual ejection are changing. The temperature range of their observation, the position of the last ejection remain unchanged. Note that in an unirradiated crystal this phenomenon does not exist. The phase transition at $180 \mathrm{~K}$ is of the first kind and is accompanied by the release of heat. The assumption that this transition is preceded by the formation of "nuclei" of a new phase allows one to explain these recombination "thermoscintillations". The heat released during the formation of "nuclei" leads to additional activation of recombination processes in the low-temperature phase.

An increase in the activation energy during the $180 \mathrm{~K}$ phase transition and the presence of the corresponding TSL peak creates a situation when it is possible to expect the appearance of a "cold" flash. Figure 6 shows this result.

After heating the crystal to the indicated temperature, recombination luminescence occurs again upon cooling. A small peak sum at $205 \mathrm{~K}$ does not allow for numerous thermal cycling.

For TSL peaks in the 260K region (Figure 4), the observed recombination luminescence has an anomalously fast luminescence flare up in the $250 \mathrm{~K}$ region. there is also a phase transition, but of the 2nd kind. The shape of the TSL curve suggests that in this case the activation energy decreases.

The spectral composition of the TSL peak at $260 \mathrm{~K}$ has the form of a single weakly asymmetric band on the long-wave side with a maximum in the region of $2.8 \mathrm{eV}$. Our measurements of the TSL curves at various doses of radiation and spectral composition on the wings of the TSL peak showed that the shape of the curve does not change qualitatively, and the radiation spectrum is the same. This suggests that this complex TSL peak is due to the decay of the same defects slightly differing in thermal stability. The crystal lattice structure of this sulfate allows the existence of one type of defects having different coordination and, therefore, thermal stability. 


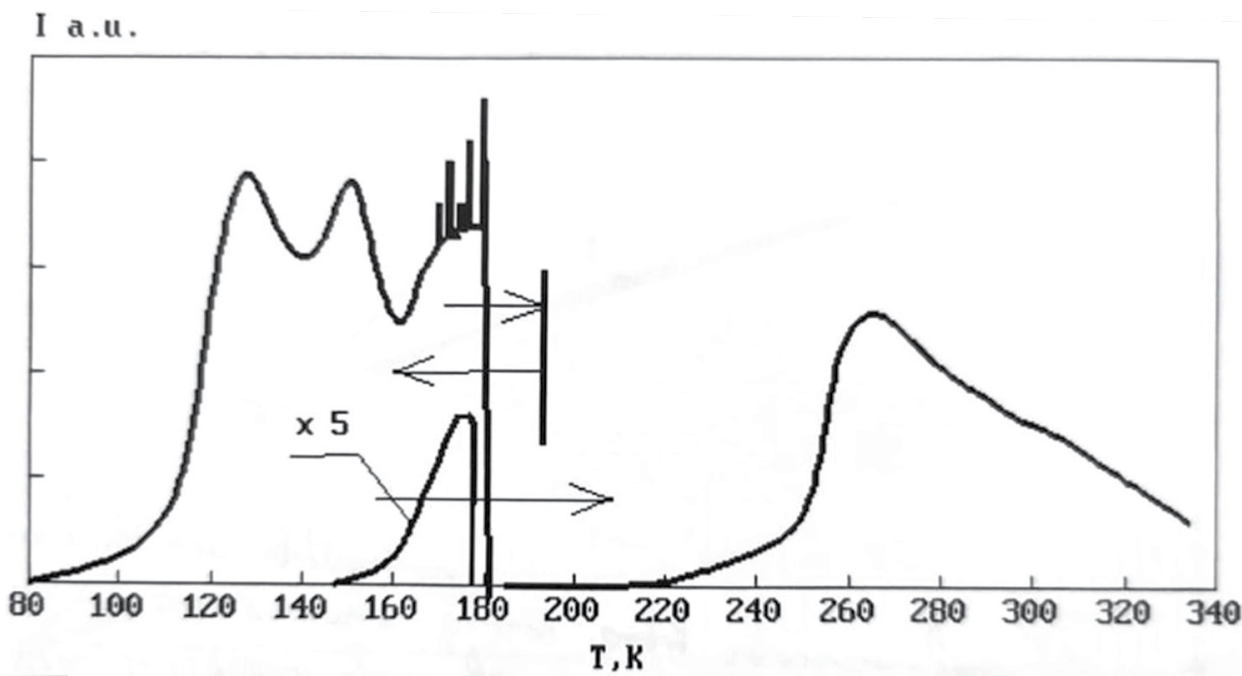

Figure 6 - "Cold flash" in $\mathrm{LiKSO}_{4}$ in the area of $170 \mathrm{~K}$. the arrows indicate the temperature course, the figure indicates the increase in gain

The TSL peak at $260 \mathrm{~K}$ does not change shape with increasing radiation dose. This allows measurements to be taken on the accumulation of the light sum depending on the irradiation temperature without separation. In figure 7 shows the dependence of the light sum in high-temperature TSL peaks on the irradiation temperature. It can be seen that above $180 \mathrm{~K}$ the nature of the dependence changes dramatically. A slight decrease in the light sum with increasing temperature is associated with a partial annealing of defects during irradiation.

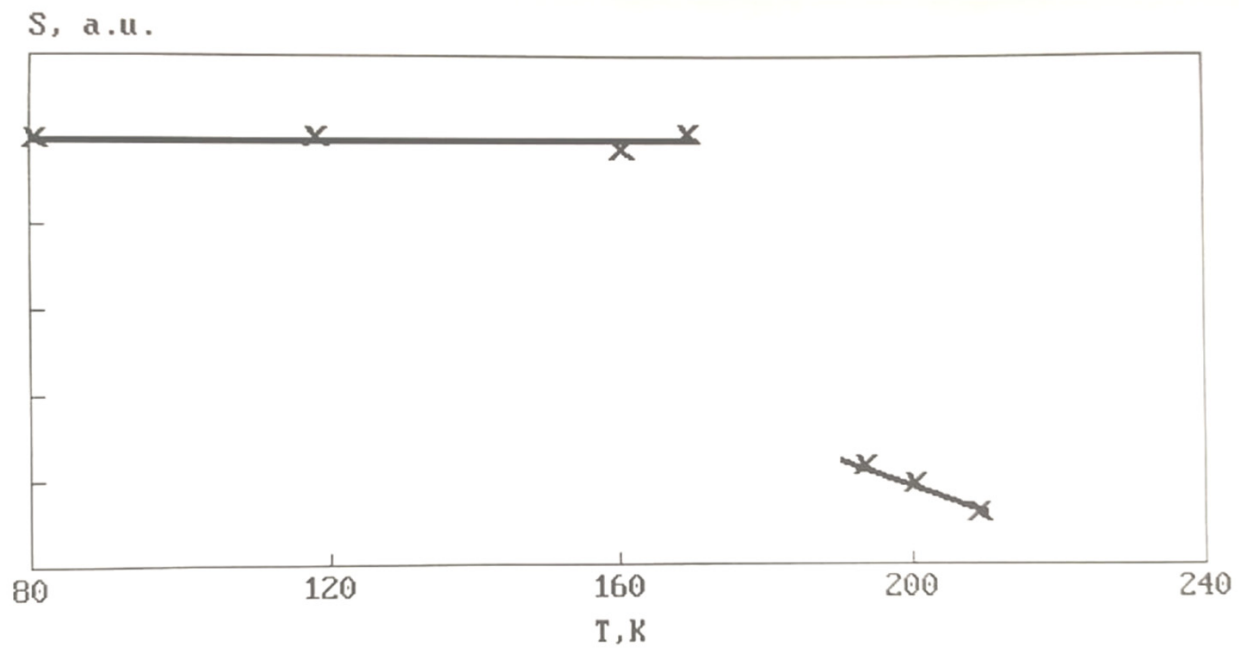

Figure 7 - Dependence of the light sum of the high-temperature TSL peaks in $\mathrm{LiKSO}_{4}$ on the irradiation temperature

Figure 8 shows the dependences of the light sum of the studied luminescence peaks on the exposure dose. After irradiation at nitrogen temperature, the accumulation curve of the light sum is well approximated by two linear dependences: with an exposure dose of up to 30 minutes and from 30 to 90 minutes (see Figure 8).

The presence of two stages of accumulation is possibly associated with the presence of 
different mechanisms of defect formation. Curve 2 shows the same dependence, but upon irradiation at a temperature of $220 \mathrm{~K}$, i.e. higher than the temperature of the first phase transition. A comparison of these curves suggests that one of the defect formation channels is structurally sensitive, while the second is not. Note that if the temperature of $220 \mathrm{~K}$ is achieved by cooling, then the light sum does not accumulate in the temperature range of $250-300 \mathrm{~K}$. The second phase transition has significant hysteresis. In cooling mode, it occurs at $200 \mathrm{~K}$. The absence of TSL peaks is due to the fact that during the irradiation process, thermal annealing of defects occurs [20-22].

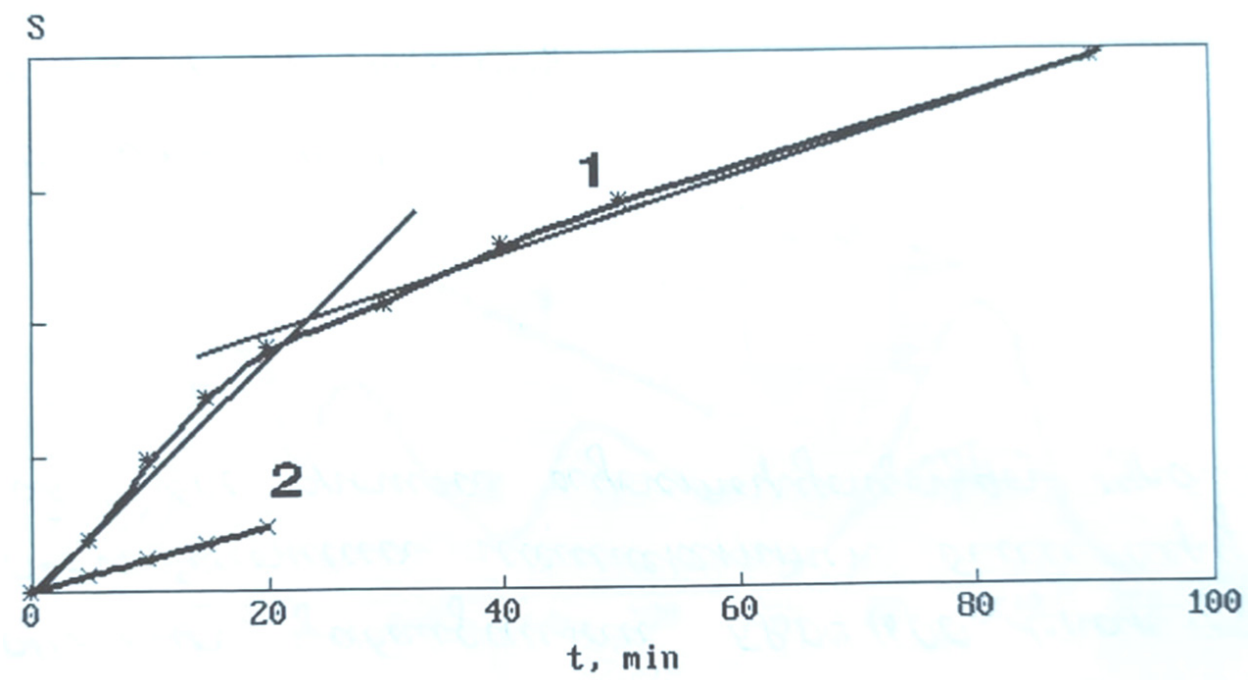

Figure 8 - Dependence of the accumulated light sum at TSL peaks in the region of $260 \mathrm{~K}$ on the exposure dose at $80 \mathrm{~K}(1)$ and $220 \mathrm{~K}(2)$ in $\mathrm{LiKSO}_{4}$

In sulfates of all known radiation defects $\left(\mathrm{SO}_{4}^{-}\right.$, $\mathrm{SO}_{3}^{-}, \mathrm{O}^{-}$and $\mathrm{SO}_{3}{ }^{2-}$ ), only $\mathrm{SO}_{3}^{-}$according to published data is formed as a result of $\mathrm{SO}_{4}^{-}$conversion [23-26].

\section{Conclusions}

We have determined by quantum chemical methods that this requires the interaction of $\mathrm{SO}_{4}^{-}$with atomic oxygen. Obviously, due to the need for a similar transformation of oxygen migration defects, the process is structurally sensitive. Another channel for the formation of $\mathrm{SO}_{3}^{-}$defects is the ionization of $\mathrm{SO}_{3}{ }^{2-}$ centers. When ammonium halide crystals are irradiated with X-rays, ionization practically does not feel changes in the lattice structure. In addition, the resulting free electron experiences dissociative capture by the sulfate anion, which leads to the formation of $\mathrm{O}^{-}$and $\mathrm{SO}_{3}^{2-}$. Consequently, ionization does not lead to a decrease in the concentration of the latter, which ensures the observed linear dependence on the radiation dose.

The above results show that the use of a polymorphic phase transition allows one to obtain additional information about radiation-stimulated processes, i.e. it can be used as a tool to study the response of crystals to ionizing radiation.

\section{References}

1. Dikke G.H., Crosswhite H.M., Dunn B. (1961). Emission Spectra of the Doubly and Triply Ionsed rare carths. J. Opt. Spec. America, 51, 820-827.

2. Aseltine C. L., Kim Y.W. (1967). Electron Paramagnetic Resonance Studies of Electron Irradiated Lithium Sulphate at Liquid Nitrogen Temperature. J. Phys. Chem. Sol., 28, 867-873.

3. Hariharan N., Sobhanandri J. (1969). ESR studies of paramagnetic centers in irradiated $\mathrm{Li}_{2} \mathrm{SO}_{4}: \mathrm{H}_{2} \mathrm{O}$. J. Phys. Chem. Sol., 30, 778-781. 
4. Kumar V.S.K., Sastry S.B.S., Acharyulu B.S. (1989). Optical stadies on Europium Doped $\mathrm{K}_{2} \mathrm{SO}_{4}$. Phys.Stat.Sol. (b), 155, 679-684.

5. Hisnnyakov V., Zazubawich S., Soovik T. (1974). Kinetik and temperatures dependens of polarised emmission of anisotropic centers in alkali halides. Phys. stat. sol., 66, 727-731.

6. Natarajan M., Secco E. (1975). Electrical Condactivity and Phase Transformation Studies on Pure and Doped $\left(\mathrm{Mg}^{+}, \mathrm{Zn}^{2+}, \mathrm{Cu}^{2+}, \mathrm{Mn}^{2+}\right)$ Crystal of $\mathrm{K}_{2} \mathrm{SO}_{4}$. Can. J. Chem., 53, 1542-1547.

7. Jia Wie-yi, Wang H., Shen J., Huang Y., Gu B.(1984). Optical rotation and phase transitions of $\mathrm{LiKSO}_{4}$ crystal. Acta phys.sin., 33, 1765-1770.

8. Wang J., Wang R., Zhang L., Li F., Lin S. (1989). The atteanuation peak and elastic anomaly of $\mathrm{LiKSO}_{4}$ crystal under low temperature. J. Low Temp. Phys., 11, 135-140.

9. Perpetuo G.I., Dantas M.S., Gazzinelli R., Pimenta M.A. (1991). Low-temperature suguence of phase trasitionin $\mathrm{LiKSO}_{4}$ studied by EPR. Phys. Rev. B, 45, 5163-5770.

10. Sheludko V.I., Nedilko S.G., Bojko V.V. (2003). Post-annealing green luminescence of sulphate crystals. Functional Matter., 10, 93-97.

11. Lochab S.P., Share P.D., Chauhan R.S., Salah N., Pandey A. (2006). Thermoluminescence and photoluminescence study of $\mathrm{Ba}_{0,97} \mathrm{Ca}_{0,03} \mathrm{SO}_{4}$ :Eu. J. Phys. D., 39, 1786-1792.

12. Su Fuhai, Ma Balei (2006). Luminescence temperature and pressure studies of $\mathrm{Zn}_{2} \mathrm{SiO}_{4}$ doped by with $\mathrm{Mn}^{3+}$ and $\mathrm{Eu}^{3+}$ ions. J. Luminescence, 116, 117-126.

13. Aitasalo T., Durigyn A., Holsa J., Lastusaari J., Suchocki A. (2004). Low temperature thermoluminescence properties of $\mathrm{Eu}^{2+}$ and $\mathrm{R}^{3+}$ doped $\mathrm{CaAl}_{2} \mathrm{O}_{4}$. Allows and compounds, 380, 4-8.

14. Lakshminarasimhan N., Varadarajui U.V.J. (2004). $\mathrm{Eu}^{3+}$ luminescence - a structural probe in $\mathrm{BiCa}_{4}\left(\mathrm{PO}_{4}\right)_{3} \mathrm{O}$ an apatite related phosphate.Solid State Chem., 177, 3536-3544.

15. Shim J.B., Yoshikawa A., Bensalah A., Fucuda T. Solovieva N., Niki M., Rosetta E., Vedda A., Toon D.H. (2003). Luminescence, radiation damage and color center creation in $\mathrm{Eu}^{3+}$-doped $\mathrm{Bi}_{4} \mathrm{Ge}_{3} \mathrm{O}_{12}$ fiber single crystals. J. Appl. Phys., 93, 5131-5135.

16. Alybakov A.A., Gubanova V.A., Kudabaev K., Sharsheev K.(1988). Optical absortion and EPR spectra of $\mathrm{Cu}^{2+}$ ions in $\mathrm{LiKSO}_{4}$ single crystals. Phys.Status.Solidi (b), 146, 135-139.

17. Kim L.M., Kuketayev T.A. (2000). Polymorphic phase transitions and recombination luminescence in crystals. Vestn. KazGU. Ser. Physical, 2 (9), 3-12.

18. Kuketayev T.A. (2003). Influence of phase transition in crystals on radiation-induced processes. 12th international conference on radiation physics and chemistry of inorganic materials. 45-46.

19. Kuketayev T.A. (2003).The effect of heterovalent impurity ions on the recombination of radiation defects in sulfates. 4th international conference "Nuclear and radiation physics", 248-249.

20. Kuketayev T.A. (2004). Radiation defect formation in lithium sulfate crystals. News of universities. Physics, 3 , $87-88$

21. Salkeyeva A.K, Kim L,M., Kuketayev T.A. (2005). Recombination luminescence of potassium sulfate activated by samarium ions. Poisk, 4, 34-40.

22. Kuketayev T.A. (1988). Radiation defects and phase transitions in crystals. Bulletin of the Academy of Sciences of the Kazakh SSR. Ser. Phys.-Math., 35-38.

23. Kuketayev T.A. (1989). Features of the decay of excitons into structural defects in crystals of ammonium halides. Solid State Physics. 6, 256-258.

24. Krupin A.S., Karyakin M.E., Molostova E.Yu. (2015). Optical properties of LC complexes of samarium (III) and europium (III). Bulletin of the Technological University, 14, 36-39.

25. Koketai T.A., Mussenova E.K. (2018). Luminescence of K2SO4 Crystals Doped by Ions of Divalent Rare-earth Elements. Book of abstracts. Functional Materials and Nanotechnologies, 164.

26. Lushchik A.Ch., Kuketay T.A., Tussupbekova A.K., Tagaeva B.S. (2015). Luminescence of potassium sulfate crystals with an admixture of ions of divalent rare earth elements. International Scientific and Practical Conference dedicated to the 90th anniversary of Academician Y.A. Buketov, 308-311. 